aktiv unterhalten. Wie eine solche aussehen kann, beschreibt Fuhrmann anhand konkreter Beispiele aus dem Bereich verbindlicher Rechtsgeschäfte und des Datenschutzrechts. Die Kritik, die er an den bestehenden rechtlichen Regelungen auf der Grundlage seiner Erkenntnisse zwangsläufig üben muss, nutzt er, um seine spannende Analyse durch rechtspolitische Vorschläge abzurunden.

Zwischen Kommunikation, Psyche, Recht, Technik und Wirtschaft existieren komplexe Verbindungen und Wechselwirkungen. Das ist keine ganz neue Erkenntnis. Sie wird aber in der aktuellen wissenschaftlichen Forschung immer noch viel zu wenig beachtet. Die vorgestellten Arbeiten gehören zu den seltenen und deshalb in ihrer Bedeutung kaum zu überschätzenden Ausnahmen. Ganz en passant räumen die Studien mit einem hartnäckigen, aber falschen Vorurteil auf: Grundlagenforschung muss nicht zwingend wertlos für die Praxis sein. Alle drei Monografien betreten nicht nur wissenschaftliches Neuland; sie beschäftigen sich mit einer Fülle von konkreten Fragen, die für die weitere Entwicklung des Electronic Commerce von erheblicher praktischer Bedeutung sind.

Volker Boehme-Neßler

\section{Julian Weiss}

\section{Das Internet und die klassischen Medien}

Konvergenz - Konkurrenz oder Komplementierung?

Eine medienpolitische Betrachtung

Frankfurt a. M. u.a.: Lang, 2003 - 396 S.

(zugl.: Mainz, Univ., Diss., 2002)

ISBN 3-631-50514-0

Die Dissertation von Julian Weiss setzt sich, so der Klappentext, das Ziel, „im Rahmen einer Gesamtbetrachtung“ „einen Beitrag zur Diskussion der rechtlichen Einordnung elektronischer Dienste in bestehende Regulierungsstrukturen zu leisten“. Und in der Einführung heißt es, dass die „strukturellen Veränderungen sowie medienpolitischen Regulierungs-Konzepte zu diskutieren und die bestehende medienrechtliche Ordnung und ihre Defizite zu untersuchen " sind. In der Tat ist dieses Buch in weiten Teilen eine umfangreiche „Gesamtbetrachtung", die sowohl technische Aspekte des
Internets, der Inhalte von Onlineangeboten traditioneller Medien, der Medienpolitik und des Medienrechts ins Visier nimmt. Allein für das erste Kapitel „Das Internet“, das Wesensmerkmale, Geschichte, technische Grundlagen und Nutzung des Internets beleuchtet, wendet Weiss 48 Seiten auf. Nicht alle Punkte, die akribisch dargestellt werden, sind allerdings für den weiteren Verlauf der Argumentation von Relevanz.

Im zweiten Kapitel stellt der Autor die Ergebnisse einer Inhaltsanalyse vor, in der die jeweils sechs reichweitenstärksten Internetangebote verschiedener Medientypen untersucht wurden: Überregionale Tageszeitungen, Publikumszeitschriften, Fachzeitschriften sowie öffentlicher wie privater Radio- und Fernsehprogramme. Weiss wertet diese Angebote hinsichtlich ihrer publizistischen Inhalte, so genannter „interaktiver Dienste“ (zu denen bereits die Möglichkeit der Suchfunktion gezählt wird), Werbung und E-Commerce aus und kommt beispielsweise bezogen auf das Fernsehen zu dem Schluss, dass sich die „vielfach angekündigte Konvergenz von Fernsehen und Internet unter inhaltlich-funktionalen Aspekten nur langsam und sporadisch entwickelt" (S. 69). Weiss vergleicht seine Befunde mit denen anderer Studien, zieht aber kein eigentliches Fazit aus ihnen. Denn was bedeutet die „langsame und sporadische" Entwicklung der Konvergenz für die Medienpolitik? Ist der Befund eine Art Entwarnung, dass die Anpassung des medienrechtlichen Rahmens doch kein allzu dringendes Problem darstellt?

Stärker theoretisch wird der Begriff der Konvergenz im dritten Kapitel definiert und diskutiert. Weiss erläutert zunächst die im Titel der Arbeit genannten Begriffe der Konkurrenz und Komplementierung und differenziert den Konvergenzbegriff dann weiter in seine technischen, inhaltlich-funktionalen und ökonomischen Aspekte.

Das vierte Kapitel wendet sich dann der Medienpolitik zu. Unter der Überschrift „Medienpolitische Grundsätze und Konfliktfelder im Zeichen der Konvergenz" wird zunächst der Begriff der Medienpolitik definiert, vom Begriff der „Kommunikationspolitik“ abgegrenzt sowie in groben Zügen die normativen Grundprinzipien der deutschen Medienordnung nachgezeichnet. Eigentlich wird erst hier die zentrale Problemstellung der Arbeit benannt: Traditionelle ordnungspolitische Grenzzie- 
hungen verlieren durch die mögliche Konvergenz an Bedeutung, neue Dienste müssen in den Regulierungsrahmen integriert werden. Oder aber man zieht aus der Schwierigkeit, die die Setzung eines neuen Regulierungsrahmens mit sich bringt, den Schluss, im Sinne einer Deregulierung ganz auf ihn zu verzichten.

Weiss greift diese Fragen im fünften Kapitel auf, in dem er die relevanten Akteure des Politikfeldes Medienpolitik, wie politisch-administrative Institutionen, gesellschaftliche und wirtschaftliche Interessengruppen sowie die öffentlichen Rundfunkanbieter, benennt und zentrale medienpolitische Regulierungskonzepte auswertet. Auffällig ist hierbei, dass die ausgewerteten Dokumente nur zum Teil von den zuvor genannten Akteuren stammen. Offenbar, so ließe sich schließen, stammen die relevanten Regulierungskonzepte mit Bezug auf die Konvergenz nicht von den traditionellen medienpolitischen Akteuren, sondern sehr stark aus dem wissenschaftlichen Bereich.

Nach diesen medienpolitischen Betrachtungen sind die nächsten drei Kapitel medienrechtlicher Natur. Weiss diskutiert die Abgrenzungsprobleme zwischen Internet und klassischen Medien zunächst unter rundfunkrechtlichen (Kapitel 6), presserechtlichen (Kapitel 7) sowie unter dem Aspekt des Europäischen Gemeinschaftsrechts (Kapitel 8). Zum Teil geht Weiss in diesen Darstellungen - im Sinne des Ziels einer "Gesamtbetrachtung" - sehr ins Detail, arbeitet die vorliegende Literatur aber soweit der Rezensent dies als Nicht-Jurist beurteilen kann - gründlich auf.

Im neunten Kapitel fasst Weiss die Ergebnisse der einzelnen bisherigen Kapitel nacheinander zusammen. Ein Zwischenfazit am Ende jedes Kapitels wäre für die Leser angenehmer gewesen, denn allzu oft wandte man sich mit einem „und was folgt daraus?" im Kopf dem nächsten Thema zu.

In der - dann mit vier Seiten doch relativ kurzen - Schlussfolgerung in Kapitel 10 greift Weiss dann die Frage nach der „Konkurrenz oder Komplementierung" wieder auf. Er betont zunächst, dass „entgegen vieler Prognosen und Annahmen [...] weder das gedruckte Wort in der Tageszeitung noch die audiovisuelle Darbietung über den Fernseher durch das Internet verdrängt oder substituiert werde" (S. 324), sondern neue Formen von Angeboten mit stärker individualkommunikativem Charakter entstanden seien. Medienpolitisch fol- gert Weiss daraus: „Die strittige Kompetenzproblematik sowie die daraus folgende Zersplitterung der Aufsichtsstrukturen führen in der praktischen Betrachtung zu dem Ergebnis, dass eine vernünftige, koordinierte Regulierung, Aufsicht und Kontrolle nicht stattfindet. Weder sind die Landesbehörden institutionell und personell in der Lage, die Vielfalt im Internet zu beaufsichtigen, noch ist die Koordination zwischen den genannten Behörden mangels institutionalisierter Abstimmung gegeben “ (S. 326). Dieses Fazit verwundert, denn Weiss hat weder die personelle Ausstattung der Landesbehörden noch die Abstimmungsprozesse zwischen ihnen untersucht - sie werden in Kapitel 5.1 nicht einmal als „Träger der Medienpolitik“ benannt. Auch fragt man sich unwillkürlich, was denn eine „vernünftige“ Regulierung sei und welche normativen Indikatoren der Autor hier heranzieht, wird aber enttäuscht, denn es sei „nicht Aufgabe und Ziel der vorliegenden Arbeit, einen Ordnungsvorschlag zu machen" (ebd.).

Als mögliche Alternative benennt Weiss dann aber doch noch, „Lösungen auf europäischer Ebene" anzustreben und einen „europäischen Ordnungsrahmen für alle IuK-Dienste“ (ebd.) zu diskutieren. Das Ziel, das Weiss mit diesem Vorschlag verbindet, ist hoch: „Im Rahmen des europäischen Integrations- und Harmonierungsprozesses sollte das Internet - insbesondere die publizistischen Angebote - als Chance begriffen werden, den Einigungsprozess voranzubringen" (S. 327). Dann aber hätte die EU nicht nur hinsichtlich ihres Gemeinschaftsrechts, sondern auch (medien-)politisch analysiert werden müssen. Im bereits erwähnten Abschnitt „Träger der Medienpolitik“ werden keine europäischen Akteure genannt, und eine ausführliche Diskussion der Frage, ob die EU politisch überhaupt willens und in der Lage ist, einen Ordnungsrahmen für alle IuK-Dienste zu schaffen, hätte diesem Vorschlag schon vorausgehen müssen. Die Auseinandersetzungen um die Zuständigkeiten der EU für den Rundfunk lassen vermuten, dass dies nicht so einfach sein dürfte. Denn auch im Rundfunkbereich wird von einigen medienpolitischen Akteuren der europäische Ordnungsrahmen ja immer dann eingefordert, wenn man eigentlich keinen haben will.

Patrick Donges 